\title{
The Ability of a Cytomegalovirus ELISPOT Assay to Predict Outcome of Low-Level CMV Reactivation in Hematopoietic Cell Transplant Recipients
}

\author{
Lynn El Haddad, ${ }^{1}$ Ella Ariza-Heredia,' Dimpy P. Shah, ${ }^{1}$ Ying Jiang, ${ }^{1}$ Ted Blanchard, ${ }^{3}$ Shashank S. Ghantoji, ${ }^{1}$ Firas El Chaer, ${ }^{1}$ Danielle El-Haddad,
} Amrita Prayag, ${ }^{1}$ Lior Nesher, ${ }^{1}$ Katy Rezvani, ${ }^{2}$ Elizabeth Shpall, ${ }^{2}$ and Roy F. Chemaly ${ }^{1}$

${ }^{1}$ Department of Infectious Diseases, Infection Control, and Employee Health, and ${ }^{2}$ Department of Stem Cell Transplantation and Cellular Therapy, The University of Texas MD Anderson Cancer Center, Houston; and ${ }^{3}$ Oxford Immunotec Incorporation, Marlborough, Massachusetts

Background. Cytomegalovirus (CMV) infections in hematopoietic cell transplant (HCT) recipients cause substantial morbidity and mortality. CMV cell-mediated immunity (CMV-CMI) can be determined by levels of interferon gamma (IFN- $\gamma$ ) production using an enzyme-linked immunospot (ELISPOT) CMV assay (T-SPOT.CMV assay). In this study, we evaluated the ability of this assay to predict the outcome of low-level CMV reactivation in HCT recipients.

Methods. We followed 55 HCT recipients with low-level CMV reactivation up to 8 weeks from enrollment. Progression to clinically significant CMV infection (CS-CMVi) was defined as a CMV load $>1000 \mathrm{IU} / \mathrm{mL}$ or $>500 \mathrm{IU} / \mathrm{mL}$ in patients receiving matched related/autologous or matched unrelated transplants, respectively, and initiation of antiviral treatment.

Results. Progression to CS-CMVi occurred in 31 (56\%) of the HCT recipients. Spot counts of CMV-specific pp65 and IE1 antigens were significantly lower in patients who had CS-CMVi than in patients who did not. On multivariate analysis, the ELISPOT $\mathrm{CMV}$ responses and steroids use were the only predictors of progression to CS-CMVi.

Conclusions. A strong association between low CMV-CMI and progression to CS-CMVi was observed in HCT recipients. The implementation of serial monitoring of CMV-CMI may identify patients at risk of progression to CS-CMVi that require antiviral therapy.

Keywords. cytomegalovirus; cell-mediated immunity; ELISPOT assay; low-level CMV reactivation; hematopoietic stem cell transplant.

Cytomegalovirus (CMV) infection may lead to life-threatening complications in immunocompromised individuals, including recipients of hematopoietic cell transplantation (HCT) [1]. In particular, patients who are CMV seropositive (IgG positive) are considered to be at high risk for CMV reactivation $[2,3]$. Around 5\% of allogeneic HCT (allo-HCT) recipients who are CMV seropositive develop CMV end-organ disease $[4,5]$. In some studies, CMV infections were associated with graft failure, graft-versus-host disease (GVHD), and secondary bacterial and fungal infections after transplantation $[6,7]$.

Preemptive therapy has been the main strategy employed for at least a decade for prevention of CMV end-organ disease. It consists of initiating anti-CMV drugs once CMV is detected at a certain threshold in whole blood or plasma of allo-HCT recipients [8]. The specific threshold for therapy initiation is not well

Received 12 July 2018; editorial decision 27 September 2018; accepted 4 October 2018; published online October 6, 2018.

Correspondence: R. F. Chemaly, MD, MPH, Department of Infectious Diseases, Infection Control, and Employee Health, The University of Texas MD Anderson Cancer Center, 1515 Holcombe Boulevard, Unit 1460, Houston, TX 77030-4095 (rfchemaly@mdanderson.org).

The Journal of Infectious Diseases ${ }^{\circledR} \quad$ 2019;219:898-907

(C) The Author(s) 2018. Published by Oxford University Press for the Infectious Diseases Society of America. All rights reserved. For permissions, e-mail: journals.permissions@oup.com. DOI: 10.1093/infdis/jiy592 defined and is center and host specific. Considering the toxic effects of the available antiviral agents, this strategy could be detrimental for patients deemed at low risk for CMV end-organ disease and with CMV load $<1000 \mathrm{IU} / \mathrm{mL}$ [8].

CMV cell-mediated immunity (CMV-CMI) is essential to control CMV replication and prevent progression to CMV end-organ disease in patients with prior exposure $[9,10]$. CMV-CMI is assessed by the interaction of both $\mathrm{CD} 4^{+}$and $\mathrm{CD} 8^{+} \mathrm{T}$ cells and $\mathrm{CD}^{+} \mathrm{T}$-cell production of interferon gamma (IFN- $\gamma$ ) and other cytokines in the presence of CMV. The enzyme-linked immunospot (ELISPOT) CMV assay (T-SPOT.CMV assay; Oxford Diagnostic Laboratories, Memphis, TN), allows the evaluation of $\mathrm{CD}^{+}$and $\mathrm{CD} 8^{+} \mathrm{T}$-cell immunity by an ex vivo stimulation of both $\mathrm{CD}^{+}$and $\mathrm{CD}^{+} \mathrm{T}$ cells with $\mathrm{CMV}$ antigens reacting with human leukocyte antigen class I haplotypes, thus triggering the production and release of IFN- $\gamma$. Patients with latent CMV infection have $\mathrm{CD}^{+}$and $\mathrm{CD} 8^{+} \mathrm{T}$ cells that recognize these antigens and secrete IFN- $\gamma$ in response, and detecting this process by a modified version of the traditional enzyme-linked immunosorbent assay (ELISA) forms the basis of the assay $[11,12]$. The utility of this ELISPOT CMV assay has been studied in the solid-organ transplant (SOT) population $[13,14]$. In a study of heart transplant recipients, it was shown that early identified high responders (spot count [SPC] >100 per 250000 cells) maintained a high 
immunity level during the first 100 days after transplantation and were protected against CMV infections, whereas low responders (SPC $<50$ per 250000 cells) experienced a higher incidence of CMV infections [13]. The same observation was noted in lung transplant recipients [14]. Recently, we evaluated the feasibility of this assay with allo-HCT recipients and showed that patients with high CMV T-cell responses were protected from developing clinically significant CMV infection (CS-CMVi) [12]. However, factors such as the occurrence of GVHD and the use of systemic corticosteroids favored the development of CMV reactivation, regardless of the ELISPOT CMV assay result. In addition, measuring CMV-CMI to ascertain the outcome of low-level CMV reactivation and patients who are at risk for progression to either high level of CMV load or end-organ disease still needs to be investigated. The purpose of the study was to determine the ability of this ELISPOT CMV assay to predict the outcome of low-level CMV reactivation in terms of progression to high CMV load and/ or CMV end-organ disease necessitating antiviral therapy.

\section{METHODS}

\section{Study Design}

During this prospective observational cohort study, 55 CMVseropositive adults who had undergone HCT between October 2015 and November 2016 (matched related, matched unrelated, or autologous) and developed low-level CMV reactivation after transplant were enrolled. Low-level reactivation was defined as CMV load $<1000 \mathrm{IU} / \mathrm{mL}$ or $<500 \mathrm{IU} / \mathrm{mL}$ (if patients had GVHD or were receiving systemic corticosteroids at the time of enrollment) and not necessitating anti-CMV therapy. Patients with a known prior CMV reactivation or CMV end-organ disease as well as patients on antiviral therapy active against CMV were excluded. Patients undergoing haploidentical and cord blood transplantation were excluded as they constitute a high-risk population and are more likely to experience CS-CMVi and disease than those receiving transplantation from matched related and unrelated donors [15]. Patients were monitored weekly using the ELISPOT CMV assay up to 8 weeks (60 days) from the date of enrollment. The study was approved by The University of Texas MD Anderson Cancer Center Institutional Review Board, and written informed consent was obtained from each participant.

\section{Definitions}

The primary end point of the study was the progression from low level of reactivation to a CS-CMVi defined as a minimum of 50\% increase in CMV load in the blood and/or the onset of CMV end-organ disease within 60 days of enrollment, and the subsequent initiation of CMV antiviral therapy by the treating physician in accordance with our institutional guidelines [16]. This end point was defined as a high CMV load of $>1000$ IU/ $\mathrm{mL}$ in low-risk patients via polymerase chain reaction (PCR) (without active GVHD and/or on systemic corticosteroid treatment within 60 days of enrollment) or a high CMV load of $>500 \mathrm{IU} / \mathrm{mL}$ in high-risk patients (with active GVHD and/ or on systemic corticosteroids within 60 days of enrollment). Low and high doses of systemic corticosteroids were defined as the administration of less or more than $600 \mathrm{mg}$ of prednisolone or equivalent per week, respectively. The secondary end points were cancer relapse, CMV end-organ disease, and all-cause mortality [17], up to 1 year from enrollment. CMV reactivation and CMV end-organ disease were defined as per Ljungman et al's updated definitions published in 2017 [17].

\section{Laboratory Analyses}

Patients had blood samples drawn upon enrollment, and afterwards every week for the next 8 weeks. The deidentified samples were shipped on the same day to Oxford Diagnostic Laboratories where the ELISPOT CMV assay was performed within 32 hours after collection, in accordance with validated test procedures. T-cell immune activity was assessed by detecting the production of IFN- $\gamma$ following ex vivo stimulation with CMV antigens (immediate early 1 [IE-1] and phosphoprotein 65 [pp65]). Results were reported in SPC per 250000 cells. The nil control SPC were subtracted from the SPC obtained for IE- 1 and pp65 if the nil control SPC were $\leq 10$. The results were not reported if (1) cell counts were less than 75000 cells per well, that is insufficient cell count, and/or (2) if the nil control SPC were $>10$. The results of the assays were not communicated in real time as they were performed in batches by the sponsor, thus, were not made available to the treating physician. No patients' characteristics nor outcomes were shared with the sponsor. Patients were managed as per standard of care and based on guidance set forth in the protocol; patients received preemptive antiviral therapy (ganciclovir or foscarnet) once the CMV load in the blood exceeded a defined threshold [18]. Additionally, CMV load by PCR was performed at least once a week using the Roche Cobas platform (lower limit of detection of $137 \mathrm{IU} / \mathrm{mL}$ ) and all results (IU/mL) from enrollment to week 8 were analyzed in this study.

\section{Patient Characteristics and Outcomes Collection}

Data on clinical characteristics were collected for each patient, including age, sex, race, type of underlying malignancy, type of transplant, and donor CMV serostatus. Additional data collected included presence of GVHD, corticosteroids use, and CMV management. Data on investigations performed if CMV end-organ disease was suspected, such as bronchoalveolar lavage and colonoscopy, as well as pathology results for biopsy samples, were obtained. Study data were managed using REDCap electronic data capture tools [19]. Moreover, the 3 following outcomes were recorded up to 1 year from enrollment: cancer relapse, $\mathrm{CMV}$ end-organ disease, and all-cause mortality.

\section{Statistical Analyses}

Optimal cutoffs for primary end-point analysis were 50 SPC per 250000 cells for IE antigen and 100 SPC per 250000 cells for pp65 antigen as previously reported by Nesher et al [12]. 
Patients were classified into 2 categories: those with a high response to the ELISPOT CMV assay, hereto high CMV-CMI (if the SPC of either antigen was above the aforementioned thresholds; above 50 for IE1 antigen and/or above 100 for pp65 antigen) and those with a low response to the ELISPOT CMV assay, hereto low CMV-CMI (if the SPC of both antigens were below the aforementioned threshold). For patients with a CMV event, CMV-specific pp65 and IE1 SPC preceding the event by 1 to 2 weeks were considered. For patients who did not experience a CMV event, the mean SPC of pp65 and IE1 over the 8-week study period for each patient was used to distinguish low and high CMV-CMI. Sample size calculation was performed for this prospective study. Assuming a CMV progression rate of $\geq 50 \%$ in patients with low CMV-CMI and a rate of $\leq 8 \%$ in those with a high CMV-CMI, a sample size of 56 patients would yield $90 \%$ power to detect a significant difference in CMV progression rate between patients with low and high responses. Considering potential early dropouts, we increased patients' enrollment by $10 \%$.

The demographic, clinical, and laboratory characteristics were compared between patients who progressed to CS-CMVi and those who did not. Continuous variables were compared using the Wilcoxon rank sum test. Categorical variables were compared using the $X^{2}$ or Fisher exact test. The Cox proportional hazards regression method was used to identify independent predictors of CMV progression. Steroid use and CMV-CMI (low/high) were time-dependent variables in the Cox regression analysis as they both changed over time during the study period. The cumulative incidence curves of CMV progression were estimated for patients with different ELISPOT CMV responses using the Simon and Makuch method [20], and a univariate Cox regression analysis was performed for the comparison. Similarly, CMV-CMI was a time-dependent variable in these analyses. The method of generalized estimating equations was used to assess the effect of decline of pp65 or IE1 SPCs on CMV progression. The predictive performance of the ELISPOT CMV assay for CMV progression was evaluated. Correlation between CMV load and pp65 and IE1 over the 8-week study period were assessed using the Spearman correlation coefficient. All tests were 2-sided with a significance level of 0.05. Statistical analyses were performed using SAS version 9.3 (SAS Institute Inc., Cary, NC) and STATA version 13 (STATACorp, College Station, TX) softwares.

\section{RESULTS}

\section{Patient Characteristics}

Sixty-two patients were enrolled in this study between October 2015 and November 2016. Seven patients were excluded with 2 withdrawals, 3 screen failures, and 2 patients with no local CMV load. In total, $55 \mathrm{HCT}$ recipients were monitored for 8 weeks or 60 days from enrollment (Figure 1). Table 1 depicts the

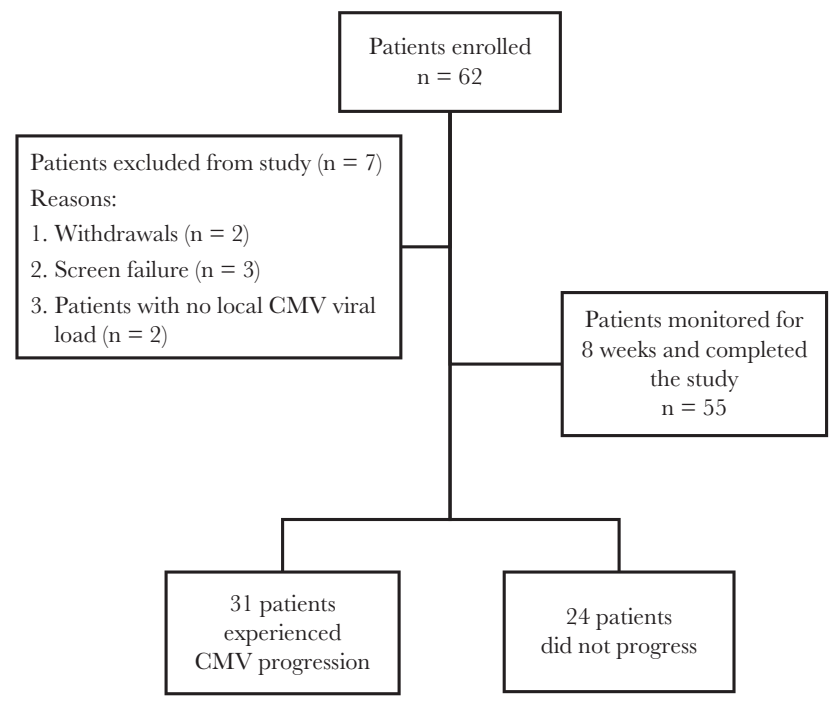

Figure 1. Flow diagram for patients' enrollment and cytomegalovirus (CMV) progression.

characteristics and outcomes of patients with or without CMV progression. Patients who received transplants from matched unrelated donors were more likely to experience CMV progression over the 60-day study period than were patients who had matched related and autologous HCT (relative risk $[\mathrm{RR}]=2.5$; 95\% confidence interval $[\mathrm{CI}], 1.5-4.3 ; P=.002)$. Patients who received $\mathrm{HCT}$ from a $\mathrm{CMV}$-seronegative donor were 2 times more likely to have CMV progression $(\mathrm{RR}=2$; 95\% CI, 1.23.6; $P=.005$ ) than those who received HCT from a CMVseropositive donor. The median peak CMV load for patients who experienced CMV progression was $3026 \mathrm{IU} / \mathrm{mL}$, with a maximum of $153456 \mathrm{IU} / \mathrm{mL}$, compared to a median peak CMV load of less than the detectable $137 \mathrm{IU} / \mathrm{mL}$ limit for those who did not progress to CS-CMVi $(P<.0001)$.

\section{Responses to the CMV ELISPOT Assay and Outcomes}

The CMV-CMI results with high background nil control (nil control > 10) were not reported. It occurred in 4 out of the total 427 samples tested, bringing the indeterminate rate to $0.9 \%$. Progression to CS-CMVi occurred in 31 (56\%) of HCT recipients. Among the 31 patients who progressed to CS-CMVi, 29 had low CMV-CMI. The remaining 2 patients who had high CMV-CMI and CS-CMVi, were diagnosed with gastrointestinal GVHD at week 5 and acute skin GVHD at week 2 after enrollment, respectively, and both received systemic corticosteroids. Furthermore, HCT recipients who had a low CMV-CMI were 8.3 times more likely to experience CMV progression than those with a high CMV-CMI (RR $=8.3$; 95\% CI, 2.2-31.1; $P<.0001)$ (Table 1). On the other hand, $75 \%$ of those who did not have CMV progression had high CMV-CMI $(P<.0001)$. In addition, $52 \%$ of the patients who had CS-CMVi were on steroids ( $63 \%$ were on $>600 \mathrm{mg}$ of prednisolone or equivalent per week) 


\begin{tabular}{|c|c|c|c|c|c|}
\hline Characteristic & $\begin{array}{c}\text { Total } \\
(n=55)\end{array}$ & $\begin{array}{l}\text { Progression to CS-CMVi } \\
\qquad(n=31)\end{array}$ & $\begin{array}{l}\text { No progression } \\
\quad(n=24)\end{array}$ & $P$ Value & $\begin{array}{l}\text { Relative Risk } \\
(95 \% \mathrm{Cl})^{\mathrm{a}}\end{array}$ \\
\hline Age, median y (range) & $60(18-73)$ & $60(18-73)$ & $58(26-68)$ & .85 & $\ldots$ \\
\hline \multicolumn{6}{|l|}{ Sex } \\
\hline Male & $31(56)$ & $14(45)$ & $17(71)$ & .06 & $\ldots$ \\
\hline Female & $24(44)$ & $17(55)$ & $7(29)$ & & \\
\hline \multicolumn{6}{|l|}{ Race } \\
\hline White & $38(69)$ & $23(74)$ & $15(63)$ & .84 & $\ldots$ \\
\hline African American & $2(4)$ & $1(3)$ & $1(4)$ & & \\
\hline Hispanic & $5(9)$ & $2(6)$ & $3(13)$ & & \\
\hline Other & $10(18)$ & $5(16)$ & $5(21)$ & & \\
\hline \multicolumn{6}{|l|}{ Type of cancer } \\
\hline Leukemia & $39(71)$ & $25(81)$ & $14(58)$ & .13 & \\
\hline Lymphoma & $8(15)$ & $2(6)$ & $6(25)$ & & \\
\hline Other & $8(15)$ & $4(13)$ & $4(17)$ & & \\
\hline \multicolumn{6}{|l|}{ Type of transplant } \\
\hline Matched related donor & $15(27)$ & $3(10)$ & $12(50)$ & .002 & Reference \\
\hline Matched unrelated donor & $38(69)$ & $26(84)$ & $12(50)$ & & $2.5(1.5-4.3)$ \\
\hline Autologous & $2(4)$ & $2(6)$ & 0 & & \\
\hline Baseline corticosteroid use & $25(45)$ & $15(48)$ & $10(42)$ & .62 & $\ldots$ \\
\hline Baseline GVHD & $11(20)$ & $6(19)$ & $5(21)$ & $>.99$ & $\ldots$ \\
\hline \multicolumn{6}{|l|}{ HCT donor status } \\
\hline CMV- & $30(55)$ & $22(71)$ & $8(33)$ & .005 & $2.0(1.2-3.6)$ \\
\hline $\mathrm{CMV}+$ & $25(45)$ & $9(29)$ & $16(67)$ & & Reference \\
\hline Time from HCT to enrollment, median d (range) & $22(2-56)$ & $21(2-51)$ & $27(4-56)$ & .28 & $\ldots$ \\
\hline Peak CMV PCR (IU/mL), median (range) & $790(137-153456)$ & $3026(137-153456)$ & $137(137-790)$ & $<.0001$ & \\
\hline Preemptive therapy against CMV & $31(56)$ & $30(97)$ & $1(4)^{\mathrm{b}}$ & $<.0001$ & $29.7(4.3-204.7)$ \\
\hline CMV pneumonia (up to 1 y from $\mathrm{HCT}$ ) & $5(9)$ & $5(17)$ & $\ldots$ & $\ldots$ & $\ldots$ \\
\hline All-cause mortality (up to 1 y) & $18(33)$ & $12(39)$ & $6(25)$ & .28 & $\ldots$ \\
\hline \multicolumn{6}{|l|}{ CMV-CMI } \\
\hline High & $20(36)$ & $2(6)$ & $18(75)$ & $<.0001$ & Reference \\
\hline Low & $35(64)$ & $29(94)$ & $6(25)$ & & $8.3(2.2-31.1)$ \\
\hline
\end{tabular}

High CMV-CMI was defined as a pp65 SPC of $>100$ per 250000 cells and/or an IE1 SPC of $>50$ per 250000 cells. Low CMV-CMI was defined as a pp65 SPC of $\leq 100$ per 250000 cells and an IE1 of $\leq 50$ SPC per 250000 cells. Values indicate median (\%).

Abbreviations: Cl, confidence interval; CMV, cytomegalovirus; CMV-CMI, cytomegalovirus cell mediated immunity; CS-CMVi, clinically significant CMV infection; GVHD, graft-versus-host disease; HCT, hematopoietic cell transplant; PCR, polymerase chain reaction; SPC, spot count.

aRelative risk was not calculated for continuous and nonsignificant variables.

bone patient was treated with valganciclovir starting at day 9 postenrollment. The patient received antiviral therapy for a maximum $\mathrm{CMV}$ load of $518 \mathrm{IU} / \mathrm{mL}$ although he was not considered in the high-risk group.

whereas only $13 \%$ of the patients who did not progress received steroids (33\% were on $>600 \mathrm{mg}$ of prednisolone or equivalent per week). Inverse correlations of peak CMV load with CMVspecific pp65 SPC, as well as with IE1 SPC, were observed (pp65 correlation coefficient $=-0.64, P<.0001$; IE1 correlation coefficient $=-0.57, P<.0001)$. The pp65 SPC was significantly lower in patients who had CMV progression (median, 37 SPC per 250000 cells; range, 0-426) when compared to patients who did not (median, 283 SPC per 250000 cells; range, 47-676; $P<.0001$ ) (Figure 2A). Another difference was encountered when comparing IE1 SPC in patients who progressed from lowlevel CMV reactivation (median, 3 SPC per 250000 cells; range, 0-254) compared to those who did not (median, 52 SPC per 250000 cells; range, 3-401; $P<.0001$ ) (Figure 2B). The level of CMV load inversely correlated with CMV-CMI. Patients who had low CMV-CMI experienced higher CMV load when compared to those with high CMV-CMI throughout the 8 week period with both IE1 and pp65 $(P<.0001)$ (Figure 3$)$.

The risk of CMV progression increased by $2 \%$ for a decline of 20 pp65 SPC per 250000 cells by the next point of measurement ( $\mathrm{RR}=1.02 ; 95 \% \mathrm{CI}, 1.003-1.03 ; P=.039)$. A similar conclusion was deduced when assessing the impact of decline of 20 IE1 SPC per 250000 cells on CMV progression $(\mathrm{RR}=1.01$; $95 \% \mathrm{CI}, 1.006$ $1.02 ; P=.002)$. The cumulative incidence curve estimates showed that a low CMV-CMI was significantly associated with CMV progression during the first 60 days after enrollment (Figure 4).

Among the 55 enrolled patients, there were 2 with proven, 2 with probable, and 1 with possible CMV pneumonitis. Two of these 5 patients had CMV disease within the 8-week study, meeting the clinical endpoint for CMV progression from lowlevel reactivation to CS-CMVi (time to CMV disease was 21 and 58 days, respectively). The remaining 3 patients experienced 
A

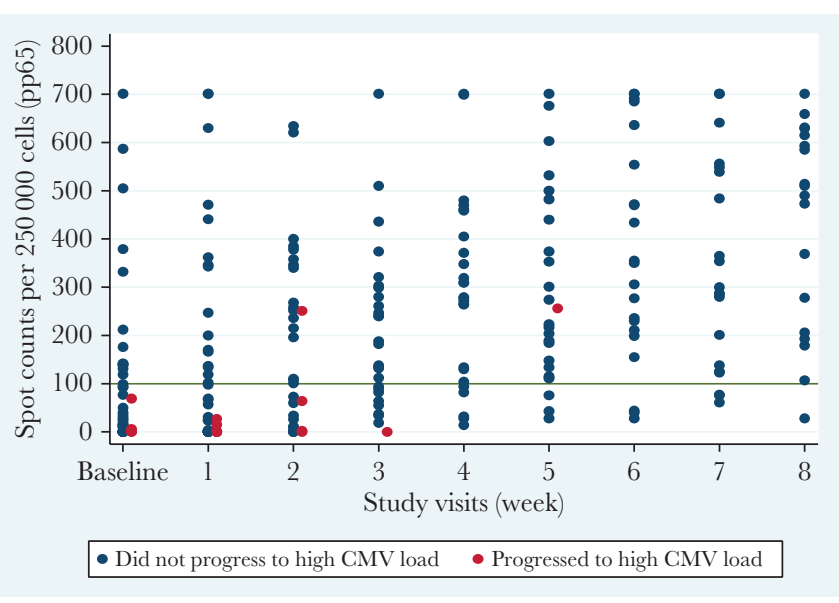

B

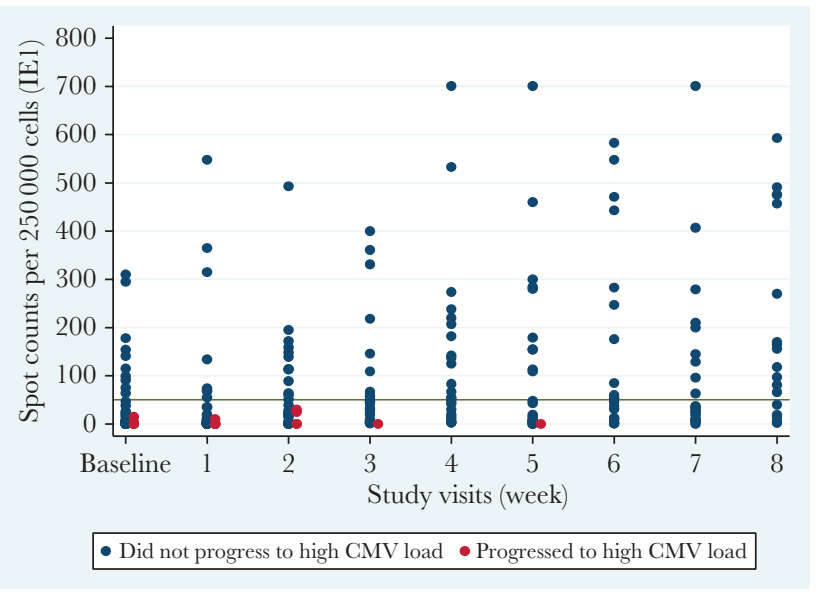

Figure 2. Scatterplots for CMV reactivation versus the number of spots produced in the ELISPOT CMV assay for pp65 $(A)$ and IE1 (B) antigens at various time points. The reference lines at 100 SPC per 250000 cells $(A)$ and at 50 SPC per 250000 cells $(B)$ denote the study-specific thresholds. Note: Patients with CMV disease were not included in this figure. Abbreviations: CMV, cytomegalovirus; ELISPOT, enzyme-linked immunospot; IE1, immediate-early protein 1; pp65, phosphoprotein 65; SPC, spot count.

CMV disease at 64, 130, and 311 days after enrollment. Out of the 18 patients $(33 \%)$ with fatal outcome within a year of study enrollment, 12 (67\%) had a CS-CMVi during the study period, including 3 (17\%) patients with CMV disease within the 1 year of follow-up. Most of the deaths were related to cancer relapse (61\%) and GVHD (11\%), and none were CMV related.

\section{Risk Factors for Progression to High CMV Load}

A Cox regression analysis identified risk factors for CMV progression from low-level reactivation. In the univariate analysis, the type of transplant $(P=.003)$, steroids use $(P<.0001)$, the donor's CMV serostatus $(P=.009)$, the level of CMV load $(P<.0001)$, and low CMV-CMI $(P<.0001)$ were associated with CMV progression. In the multivariate analysis, low CMVCMI $(P<.0001)$ and steroids use $(P=.002)$ were the only significant predictors of CMV progression (Table 2 ).

\section{Diagnostic Accuracy}

The sensitivity of the ELISPOT CMV assay as a predictor of progression to CS-CMVi in case of a low response was $94 \%$, and the negative predictive value, indicating protection against CMV progression in case of a high response, was $90 \%$.

\section{DISCUSSION}

In this study, the ELISPOT CMV assay was evaluated for its ability to measure IFN- $\gamma$ release as a marker of protection against progression of low-level CMV reactivation in HCT recipients. Our main finding was the strong correlation between high CMV-CMI and the absence of progression to CS-CMVi and/ or CMV disease. In fact, patients with high CMV-CMI were protected from developing any CS-CMVi necessitating antiviral therapy, whereas patients with low CMV-CMI were significantly more prone to progression and receiving antiviral therapy.
The CMV-CMI and steroids use were the only predictors of CMV progression after controlling for many confounders. A high negative predictive value and a high sensitivity were found in this study when using the ELISPOT CMV assay to determine protection against CMV progression from low-level reactivation. Using cutoffs of 50 SPCs per 250000 cells for IE1 and 100 SPCs per 250000 cells for pp65 determined which patients were at risk of developing CS-CMVi. High CMV-CMI against either pp65 (>100 SPCs per 250000 cells) or IE1 (>50 SPCs per 250000 cells) proteins are enough to protect patients from CS-CMVi $[12,21]$. Several studies have shown the ability of the ELISPOT CMV assay to predict CMV reactivation risks, in HCT and SOT recipients [12, 22, 23]. Other diagnostic methods have been developed in order to detect and monitor CMV-CMI. QuantiFERON-CMV assay (Melbourne, Australia) is an ELISA-based assay that is easy to perform and does not require a high volume of blood [24]. Although this assay may detect patients at risk of developing CMV infection [25-27], reduced sensitivity has been reported, especially in immunocompromised patients $[28,29]$. In SOT recipients, this assay was not predictive of CMV reactivation and was associated with higher incidence of CMV disease in high-risk SOT recipients with indeterminate results (58.3\%) [30]. Furthermore, in allogeneic HCT recipients, the sensitivity and the specificity of the QuantiFERON-CMV assay were reduced, with up to $38 \%$ indeterminate or not interpretable results [27, 31].

We found that patients receiving steroids, high doses in particular, at the time of low-level CMV reactivation were at high risk of progression to CS-CMVi. Steroids and other risk factors, such as GVHD and type of transplant, are well described predisposing factors for CMV reactivation $[1,32]$ as well as CMV end-organ disease [33] in HCT recipients. Actually, patients 

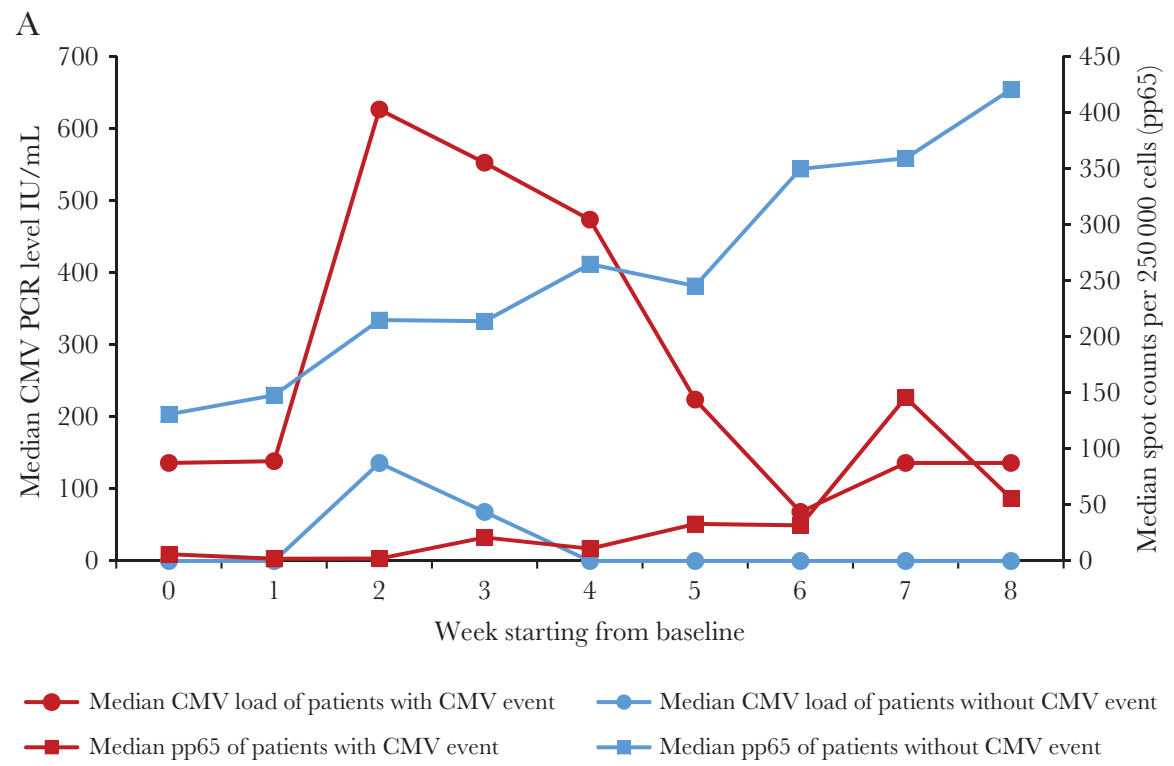

B

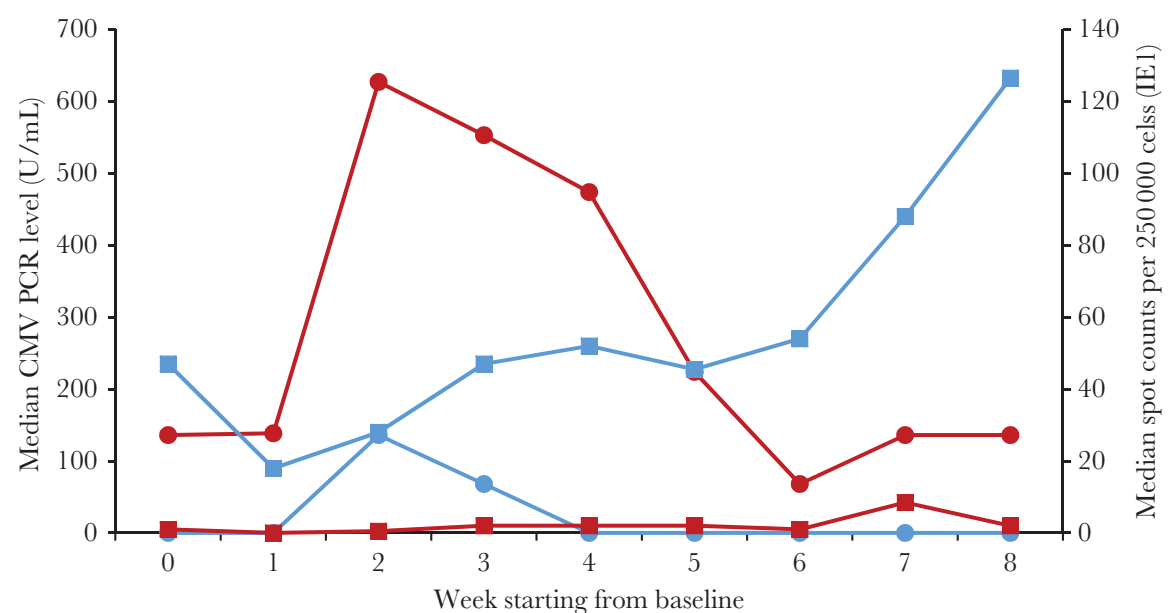

$\longrightarrow$ Median CMV load of patients with CMV event

- - Median CMV load of patients without CMV event

$\rightarrow$ Median IE1 of patients with CMV event

- Median IE1 of patients without CMV event

Figure 3. Changes in the median of pp65 SPC (A) and IE1 SPC (B) (both expressed as SPC per 250000 cells), and CMV load (expressed in international units per milliliter) over the 8-week study period in patients with or without CMV events. Abbreviations: CMV, cytomegalovirus; IE1, immediate-early protein 1; PCR, polymerase chain reaction; pp65, phosphoprotein 65; SPC, spot count.

receiving steroids were shown to have impaired and low numbers of CMV-specific cytotoxic T cells [34]. Of interest, only 2 autologous transplant recipients were included in the study as they met the eligibility criteria. When excluding these 2 patients from the cohort, the new analysis yielded the same results (data not shown) and had no bearing on our conclusions.

Peak viral load of patients who experienced CMV progression was higher than that of patients who did not. Patients with high CMV load had a low CMV-CMI and were prone to CMV progression from low-level CMV load. Avetisyan et al showed that allogeneic HCT recipients with no IFN- $\gamma$ production and receiving pretransplant myeloablative conditioning had a higher peak CMV load and weakened T-cell reconstitution compared to those with IFN- $\gamma$ production and receiving reduced-intensity conditioning, leading to an increase in CMV replication and subsequent CMV end-organ disease [35]. Some studies showed that a high CMV load in blood is the only significant risk factor for CMV disease in HCT recipients [36, 37], whereas others showed that during the first 3 months after transplant, both CMV-CMI and CMV load are important predictors of early CMV end-organ disease and survival [22, 38]. In SOT recipients, it was shown that patients with CMV load $>1500 \mathrm{IU} / \mathrm{mL}$ and absence of CMV-CMI were at risk of recurrent $\mathrm{CMV}$ infections and were diagnosed with subsequent symptomatic CMV disease [39]. 


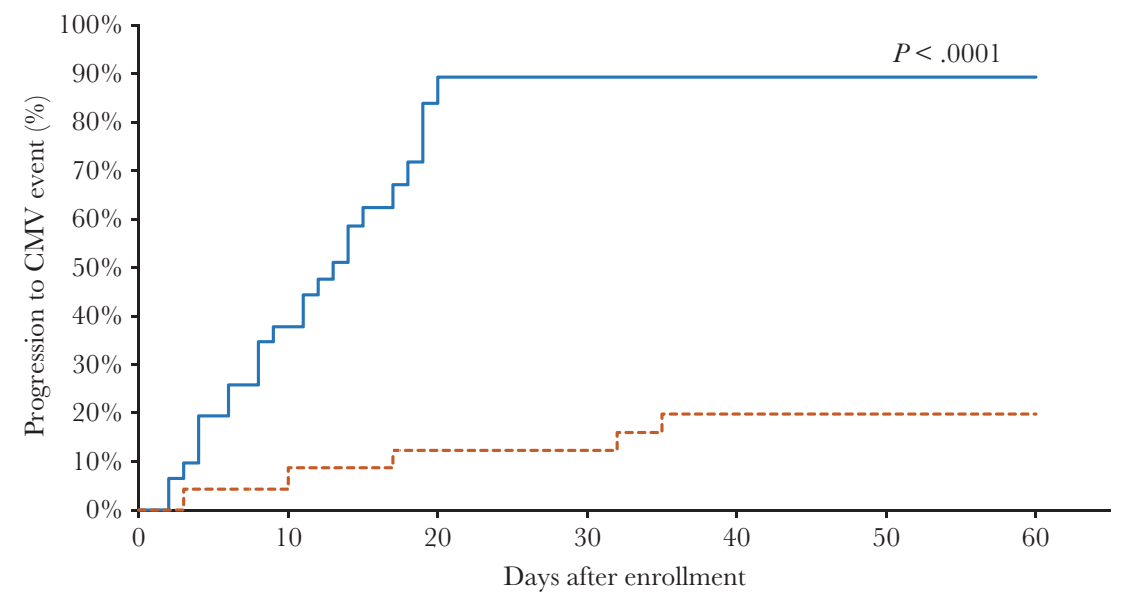

Figure 4. Cumulative incidence curves of time to clinically significant CMV infection using the Simon and Makuch method, stratified by high (dashed lines) and low responses to the ELISPOT CMV assay. Note: CMV-CMI level (low /high) was a time-dependent variable in the cumulative incidence curves as it changed over time during the study period. Abbreviations: CMV, cytomegalovirus; CMV-CMI, cytomegalovirus cell mediated immunity; ELISPOT, enzyme-linked immunospot.

\section{Table 2. Predictors of Progression to Clinically Significant CMV Infection by Cox Regression Analysis}

\begin{tabular}{|c|c|c|c|c|}
\hline \multirow[b]{2}{*}{ Variable } & \multicolumn{2}{|c|}{ Univariate Analysis } & \multicolumn{2}{|c|}{ Multivariate Analysis } \\
\hline & Crude HR (95\% Cl) & $P$ Value & Adjusted HR (95\% Cl) & $P$ Value \\
\hline Age & $0.99(0.96-1.01)$ & .32 & & \\
\hline Sex & & .12 & & $\ldots^{a}$ \\
\hline Male & Reference & & & \\
\hline Female & $1.74(0.86-3.54)$ & & & \\
\hline Race & & .89 & & \\
\hline White & Reference & & & \\
\hline African American & $0.67(0.09-4.97)$ & & & \\
\hline Hispanic & $0.64(0.15-2.70)$ & & & \\
\hline Other & $0.81(0.31-2.13)$ & & & \\
\hline Type of cancer & & .25 & & \\
\hline Leukemia & Reference & & & \\
\hline Lymphoma & $0.32(0.08-1.35)$ & & & \\
\hline Other & $0.66(0.23-1.90)$ & & & \\
\hline Type of transplant & & .003 & & $\ldots^{a}$ \\
\hline Matched related donor & Reference & & & \\
\hline Matched unrelated donor & $4.75(1.43-15.73)$ & & & \\
\hline Autologous & $24.83(3.90-158.15)$ & & & \\
\hline Steroids use ${ }^{b}$ & $5.85(2.61-13.11)$ & $<.0001$ & $4.87(2.12-11.19)$ & .002 \\
\hline GVHD & $1.39(0.65-2.96)$ & .39 & & \\
\hline HCT donor status & & .009 & & $\ldots^{a}$ \\
\hline CMV- & Reference & & & \\
\hline $\mathrm{CMV}+$ & $0.35(0.16-0.77)$ & & & \\
\hline Time from HCT to enrollment, $d$ & $0.99(0.97-1.02)$ & .47 & & \\
\hline CMV-CMI & & $<.0001$ & & $<.0001$ \\
\hline High & Reference & & Reference & \\
\hline Low & $9.68(3.49-26.80)$ & & $9.02(3.19-25.51)$ & \\
\hline
\end{tabular}

High CMV-CMI was defined as a pp65 of $>100$ per 250000 cells and/or an IE1 SPC of $>50$ per 250000 cells. Low CMV-CMI was defined as a pp65 SPC of $\leq 100$ per 250000 cells and an IE1 of $\leq 50$ SPC per 250000 cells. CMV load was excluded from the multivariate Cox regression analysis as it was highly related to the ELISPOT CMV assay results.

Abbreviations: $\mathrm{Cl}$, confidence interval; CMV, cytomegalovirus; CMV-CMI, cytomegalovirus cell mediated immunity; GVHD, graft versus host disease; HCT, hematopoietic cell transplant; HR, hazard ratio; SPC, spot count.

avariable was entered into the initial multivariate Cox regression model based on the $P$ value of its univariate analysis $(\leq .20)$ and later removed from the final Cox regression model through the backward elimination procedure.

bSteroid use and CMV-CMI level (low/high) were time-dependent variables in the Cox regression analysis as they changed over time during the study period. 
This study has few limitations. Cord blood and haploidentical transplant recipients were excluded from this study as they are more likely to experience CS-CMVi and/or disease with delay in CMV-specific T-cell recovery; therefore, generalizability of our data in these high-risk patients is inadequate. Additionally, the ELISPOT CMV assay results were not available to the treating physician in real time and did not guide therapy. Nonetheless, physicians followed a strict protocol recommended for CMV management based on risk factors and specific CMV load thresholds to initiate therapy. Future studies in HCT recipients with real-time CMV-CMI monitoring to direct management and prevention of CMV infections will be imperative as shown in a proof of concept study by Kumar et al in SOT recipients [10]. The authors monitored CMV-CMI in real time in 27 SOT recipients and found that discontinuing antiviral therapy for patients with positive CMV-CMI was significantly associated with fewer CMV recurrences [10]. Discontinuation or withholding antiviral therapy based on positive CMV-CMI may avoid unnecessary use of antiviral therapy and subsequent side effects from the commercially available anti-CMV agents (ie, ganciclovir or foscarnet) in patients at low risk for CS-CMVi or recurrence.

Recently, letermovir, a CMV terminase complex inhibitor, was approved for prevention of CS-CMVi after allogeneic HCT in adult CMV recipient seropositive, based on a phase 3 trial [16]. Its impact on low-level viremia still needs to be determined. Furthermore, the ELISPOT CMV assay may be useful in high-risk HCT recipients who may require prolonged prophylaxis with letermovir beyond day 100 .

In conclusion, our study showed an association between low CMV-CMI, steroids use, and CMV progression in HCT recipients. Monitoring patients using the ELISPOT CMV assay may help identify HCT recipients at risk of progression from low CMV levels to clinically significant CMV infection; such recipients would be monitored closely. Moreover, the implementation of this assay in clinical practice may help reduce unnecessary and/or prolonged antiviral therapy in patients who have high CMV-CMI and thus have a self-limited reactivation. Importantly, whether HCT recipients with low-level CMV reactivation and receiving steroids and/or with low CMV-CMI would have better outcomes with early antiviral therapy should be determined in future trials.

\section{Notes}

Author contributions. E. A-H., D. P. S., L. N., K. R., E. J. S., and R. F. C. designed the study. L. E., D. P. S., S. S. G., and F. E. C. enrolled patients. L. E. collected and processed all samples. L. E., D. E.-H., and A. P. collected patients' data. Y. J. and D. P. S. analyzed the data. Oxford Immunotec provided ELISPOT CMV responses and assistance with study design. E. A.-H. and L. E. supervised the study. L. E. and R. F. C. wrote and edited the manuscript.
Acknowledgments. We thank Jodi Govan and Chris Ball (Oxford Immunotec, Inc) for providing ELISPOT CMV results. We thank Sunita Patterson of the Department of Scientific Publications, MD Anderson Cancer Center, for copyediting the manuscript.

Disclaimer. The sponsor did not participate in the analysis and/or the interpretation of the data.

Financial support. This work was supported by Oxford Immunotec; and by the National Institutes of Health National Cancer Institute (grant number P30CA016672).

Potential conflicts of interest. R. F. C. has served as consultant to Oxford Immunotec, Merck, Chimerix, and Astellas, and received research funding from Oxford Immunotec, Merck, Novartis, Shire, and Chimerix. E. A.-H. has received research funding from Oxford Immunotec. T. B. is an employee of Oxford Immunotec, Inc. All other authors report no potential conflicts. All authors have submitted the ICMJE Form for Disclosure of Potential Conflicts of Interest. Conflicts that the editors consider relevant to the content of the manuscript have been disclosed.

\section{References}

1. Ljungman P, Hakki M, Boeckh M. Cytomegalovirus in hematopoietic stem cell transplant recipients. Hematol Oncol Clin North Am 2011; 25:151-69.

2. Boeckh M, Nichols WG. The impact of cytomegalovirus serostatus of donor and recipient before hematopoietic stem cell transplantation in the era of antiviral prophylaxis and preemptive therapy. Blood 2004; 103:2003-8.

3. Gandhi MK, Khanna R. Human cytomegalovirus: clinical aspects, immune regulation, and emerging treatments. Lancet Infect Dis 2004; 4:725-38.

4. Ariza-Heredia EJ, Nesher L, Chemaly RF. Cytomegalovirus diseases after hematopoietic stem cell transplantation: a mini-review. Cancer Lett 2014; 342:1-8.

5. Azevedo LS, Pierrotti LC, Abdala E, et al. Cytomegalovirus infection in transplant recipients. Clinics 2015; 70:515-23.

6. Mohty M, Faucher C, Vey N, et al. High rate of secondary viral and bacterial infections in patients undergoing allogeneic bone marrow mini-transplantation. Bone Marrow Transplant 2000; 26:251-5.

7. Nichols WG, Corey L, Gooley T, Davis C, Boeckh M. High risk of death due to bacterial and fungal infection among cytomegalovirus (CMV)-seronegative recipients of stem cell transplants from seropositive donors: evidence for indirect effects of primary CMV infection. J Infect Dis 2002; 185:273-82.

8. Boeckh M, Ljungman P. How we treat cytomegalovirus in hematopoietic cell transplant recipients. Blood 2009; 113:5711-9.

9. Egli A, Humar A, Kumar D. State-of-the-art monitoring of cytomegalovirus-specific cell-mediated immunity after 
organ transplant: a primer for the clinician. Clin Infect Dis 2012; 55:1678-89.

10. Kumar D, Mian M, Singer L, Humar A. An interventional study using cell-mediated immunity to personalize therapy for cytomegalovirus infection after transplantation. Am J Transplant 2017; 17:2468-73.

11. Barabas S, Spindler T, Kiener R, et al. An optimized IFN- $\gamma$ ELISpot assay for the sensitive and standardized monitoring of CMV protein-reactive effector cells of cell-mediated immunity. BMC Immunol 2017; 18:14.

12. Nesher L, Shah DP, Ariza-Heredia EJ, et al. Utility of the enzyme-linked immunospot interferon- $\gamma$-release assay to predict the risk of cytomegalovirus infection in hematopoietic cell transplant recipients. J Infect Dis 2016; 213:1701-7.

13. Abate D, Fiscon M, Saldan A, et al. Human cytomegalovirus-specific $\mathrm{T}$-cell immune reconstitution in preemptively treated heart transplant recipients identifies subjects at critical risk for infection. J Clin Microbiol 2012; 50:1974-80.

14. Costa C, Saldan A, Sinesi F, et al. The lack of cytomegalovirus-specific cellular immune response may contribute to the onset of organ infection and disease in lung transplant recipients. Int J Immunopathol Pharmacol 2012; 25:1003-9.

15. Montoro J, Piñana JL, Moscardó F, Sanz J. Infectious complications after umbilical cord-blood transplantation from unrelated donors. Mediterr J Hematol Infect Dis 2016; 8:e2016051.

16. Marty FM, Ljungman P, Chemaly RF, et al. Letermovir prophylaxis for cytomegalovirus in hematopoietic-cell transplantation. N Engl J Med 2017; 377:2433-44.

17. Ljungman P, Boeckh $\mathrm{M}$, Hirsch $\mathrm{HH}$, et al.; Disease Definitions Working Group of the Cytomegalovirus Drug Development Forum. Definitions of cytomegalovirus infection and disease in transplant patients for use in clinical trials. Clin Infect Dis 2017; 64:87-91.

18. Ljungman P, de la Camara R, Cordonnier C, et al.; European Conference on Infections in Leukemia. Management of CMV, HHV-6, HHV-7 and Kaposi-sarcoma herpesvirus (HHV-8) infections in patients with hematological malignancies and after SCT. Bone Marrow Transplant 2008; 42:227-40.

19. Harris PA, Taylor R, Thielke R, Payne J, Gonzalez N, Conde JG. Research electronic data capture (REDCap)-a metadata-driven methodology and workflow process for providing translational research informatics support. J Biomed Inform 2009; 42:377-81.

20. Schultz LR, Peterson EL, Breslau N. Graphing survival curve estimates for time-dependent covariates. Int J Methods Psychiatr Res 2002; 11:68-74.

21. Gyulai Z, Endresz V, Burian K, et al. Cytotoxic T lymphocyte (CTL) responses to human cytomegalovirus pp65, IE1-Exon4, gB, pp150, and pp28 in healthy individuals: reevaluation of prevalence of IE1-specific CTLs. J Infect Dis 2000; 181:1537-46.
22. Aubert G, Hassan-Walker AF, Madrigal JA, et al. Cytomegalovirus-specific cellular immune responses and viremia in recipients of allogeneic stem cell transplants. J Infect Dis 2001; 184:955-63.

23. Cantisán S, Lara R, Montejo M, et al. Pretransplant interferon- $\gamma$ secretion by CMV-specific $\mathrm{CD}^{+} \mathrm{T}$ cells informs the risk of CMV replication after transplantation. Am J Transplant 2013; 13:738-45.

24. Walker S, Fazou C, Crough T, et al. Ex vivo monitoring of human cytomegalovirus-specific $\mathrm{CD}^{+}$T-cell responses using QuantiFERON-CMV. Transpl Infect Dis 2007; 9:165-70.

25. Lisboa LF, Kumar D, Wilson LE, Humar A. Clinical utility of cytomegalovirus cell-mediated immunity in transplant recipients with cytomegalovirus viremia. Transplantation 2012; 93:195-200.

26. Lochmanova A, Lochman I, Tomaskova H, et al. Quantiferon-CMV test in prediction of cytomegalovirus infection after kidney transplantation. Transplant Proc 2010; 42:3574-7.

27. Tey SK, Kennedy GA, Cromer D, et al. Clinical assessment of anti-viral CD8 ${ }^{+} \mathrm{T}$ cell immune monitoring using QuantiFERON-CMV ${ }^{\circledast}$ assay to identify high risk allogeneic hematopoietic stem cell transplant patients with CMV infection complications. PLoS One 2013; 8:e74744.

28. Giulieri S, Manuel O. QuantiFERON ${ }^{\oplus}-\mathrm{CMV}$ assay for the assessment of cytomegalovirus cell-mediated immunity. Expert Rev Mol Diagn 2011; 11:17-25.

29. Kumar D, Chernenko S, Moussa G, et al. Cell-mediated immunity to predict cytomegalovirus disease in high-risk solid organ transplant recipients. Am J Transplant 2009; 9:1214-22.

30. Manuel O, Husain S, Kumar D, et al. Assessment of cytomegalovirus-specific cell-mediated immunity for the prediction of cytomegalovirus disease in high-risk solid-organ transplant recipients: a multicenter cohort study. Clin Infect Dis 2013; 56:817-24.

31. Clari MÁ, Muñoz-Cobo B, Solano C, et al. Performance of the QuantiFERON-cytomegalovirus (CMV) assay for detection and estimation of the magnitude and functionality of the CMV-specific gamma interferon-producing CD8(+) T-cell response in allogeneic stem cell transplant recipients. Clin Vaccine Immunol 2012; 19:791-6.

32. Camargo JF, Komanduri KV. Emerging concepts in cytomegalovirus infection following hematopoietic stem cell transplantation. Hematol Oncol Stem Cell Ther 2017; 10:233-8.

33. Emery VC, Sabin CA, Cope AV, Gor D, Hassan-Walker AF, Griffiths PD. Application of viral-load kinetics to identify patients who develop cytomegalovirus disease after transplantation. Lancet 2000; 355:2032-6. 
34. Ozdemir E, St John LS, Gillespie G, et al. Cytomegalovirus reactivation following allogeneic stem cell transplantation is associated with the presence of dysfunctional antigen-specific CD8 ${ }^{+}$T cells. Blood 2002; 100:3690-7.

35. Avetisyan G, Larsson K, Aschan J, Nilsson C, Hassan M, Ljungman P. Impact on the cytomegalovirus (CMV) viral load by CMV-specific T-cell immunity in recipients of allogeneic stem cell transplantation. Bone Marrow Transplant 2006; 38:687-92.

36. Gor D, Sabin C, Prentice HG, et al. Longitudinal fluctuations in cytomegalovirus load in bone marrow transplant patients: relationship between peak virus load, donor/ recipient serostatus, acute GVHD and CMV disease. Bone Marrow Transplant 1998; 21:597-605.
37. Meyers JD, Ljungman P, Fisher LD. Cytomegalovirus excretion as a predictor of cytomegalovirus disease after marrow transplantation: importance of cytomegalovirus viremia. J Infect Dis 1990; 162:373-80.

38. Boeckh M, Leisenring W, Riddell SR, et al. Late cytomegalovirus disease and mortality in recipients of allogeneic hematopoietic stem cell transplants: importance of viral load and T-cell immunity. Blood 2003; 101:407-14.

39. Martín-Gandul C, Pérez-Romero P, Blanco-Lobo P, et al.; Spanish Network for Research in Infectious Diseases (REIPI). Viral load, CMV-specific T-cell immune response and cytomegalovirus disease in solid organ transplant recipients at higher risk for cytomegalovirus infection during preemptive therapy. Transpl Int 2014; 27:1060-8. 